\title{
RECTIFICATION EFFECT IN GOLD/2,5- DICHLOROBENZENE-1,3-DITHIOL/GOLD METAL- MOLECULE JUNCTION-AB INITIO STUDY
}

\author{
A.Aadhityan, C. Preferencial Kala* andD. John Thiruvadigal \\ Department of Physics and Nanotechnology, Center for Materials Science and Nanodevices, \\ SRM Institute of Science and Technology, Kattankulathur, Tamil Nadu, India. \\ *E-mail: preferec@srmist.edu.in
}

\begin{abstract}
Molecular electronics bring a high speed and power consumption devices such as rectifier, negative differential resistor, transistor, etc. Rectifiers are widely used in the electronic industry as AC to DC converter. We have investigated the electron transport of Gold/2,5-dichlorobenzene-1,3-dithiol/Gold single molecular junction to study the rectification that occurs in this metal-molecular junction. To create such a molecular junction, we have sandwiched the 2,5-dichlorobenzene-1,3-dithiol molecule at the hcp site of gold. Density Functional Theory (DFT) with Non-Equilibrium Green's Function (NEGF) was used to calculate the total density of states, the projected density of states, transmission co-efficient, conductance, conductance, and current-voltage characteristic of Gold/2,5-dichlorobenzene-1,3-dithiol/Gold. From current-voltage characteristics, we found differential resistance effects present in this system. Furthermore, we have shown 2,5-dichlorobenzene-1,3-dithiol can be used as a single molecule rectifier.
\end{abstract}

Keywords: Single-molecule Rectifier, Density Functional Theory, Molecular Electronics, Differential Resistance.

(C) RASĀYAN. All rights reserved

\section{INTRODUCTION}

Metal-molecule-metal junction utilized to produce various devices such as rectifiers, switches, sensors etc. Various metal-molecule-metal junctions show a diode like behavior. ${ }^{1-6}$ Increasing the conductance of such junction is one of the emerging research in molecular electronics. Conjugated molecules show better conductance and less HOMO-LUMO gap comparing to non-conjugated molecules. ${ }^{7-11}$ Benzene is one of the simple conjugated molecules explored by many researchers. ${ }^{12,13}$

Elbing et. al. first observed the rectification effect in a molecule experimentally. ${ }^{14,15}$ Aviram A et.al studied the pi-donor, acceptor system separated by a sigma bond that acts as a molecular rectifier. ${ }^{16} \mathrm{An}$ experimental realization of molecular rectifiers using azafullerene C59N was done by Jin Zhao et al.This molecular junction produces the positive onset voltage at 0.5 to $0.7 \mathrm{~V}$ and negative onset is about 1.6-1.8 $\mathrm{V} .{ }^{17}$ Metzger RM et al.observed an electronic rectification effect in hexadecylquinolinium tricyanoquinodimethanide. ${ }^{18} \mathrm{Hihath} \mathrm{J}$ et al.studied inelastic transport and low bias rectification in a single molecular diode. ${ }^{19}$ These studies show that a single molecule can act as an electronic rectifier.

In this study, we have analyzed the rectification properties of the 2,5-dichlorobenzene-1,3-dithiol molecule. To construct metal-molecule-metal junction, we have sandwiched 2,5-dichlorobenzene-1,3dithiol molecule between gold electrodes at the hcp site. The electron will transport from one gold electrode to another electrode through this molecule. In this molecular junction, we observed a small rectification effect by changing the applied bias direction. The density of states, transmission co-efficient, conductance, and current-voltage characteristic of this device analyzed to understand the electron transport properties.

\section{Computational Details}

The Density Functional Theory (DFT) $)^{20-22}$ with Non-Equilibrium Green's Function formalism $(\mathrm{NEGF})^{23,24}$ was utilized to understand the electronic transport properties of Gold/2,5-dichlorobenzene1,3-dithiol/Gold molecular junction. Initially, this metal-molecular-metal junction was optimized using

Rasayan J. Chem., 13(3), 1412-1416(2020)

http://dx.doi.org/10.31788/RJC.2020.1333049

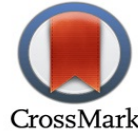


RASĀYAN J. Chem.

Vol. 13 | No. 3 |1412-1416| July - September | 2020

GGA approximation. So that the density of the electron in the system treated non-locally. To include the exchange-correlation effect we have used Perdew-Burke-Ernzerh (PBE) functional. ${ }^{25,26}$ The transport properties highly depend upon the molecule structure. Hence we have used double zeta polarized basis set for the $\mathrm{C}, \mathrm{H}$, and $\mathrm{S}$ atoms and single zeta polarized basis set for gold atoms. The electrode calculations have been performed under periodic boundary conditions, and the Brillouin zone has been sampled with $3 \times 3 \times 100 \mathrm{k}$-points on $\mathrm{x}, \mathrm{y}$, and $\mathrm{z}$-axis, where $\mathrm{z}$ is the transport direction. ${ }^{27}$ This metal-molecule-metal junction optimized until the residual force becomes less than $0.05 \mathrm{eV}$. The structure of the molecular junction is shown in Fig.-1. The optimized device structure was used to calculate the density of states (DOS), the projected density of states (PDOS), transmission co-efficient, etc. we have used ATKVNL[28] for constructing and computing this metal-molecule-metal junction. The current through this metal-molecule-metal junction was using Landauer formula,

$$
I=\frac{2 e}{h} \int T(E, V)\left[f\left(E-\mu_{L}\right)-f\left(E-\mu_{R}\right)\right] d E
$$

Where $\mathrm{f}$ is the Fermi-Dirac distribution function of electrodes. $\mu_{\mathrm{L}}$ and $\mu_{\mathrm{R}}$ represent the left and right electrode potentials. ${ }^{29}$ The rectification ratio of these devices has calculated by using the computed current between forward and reverse bias.

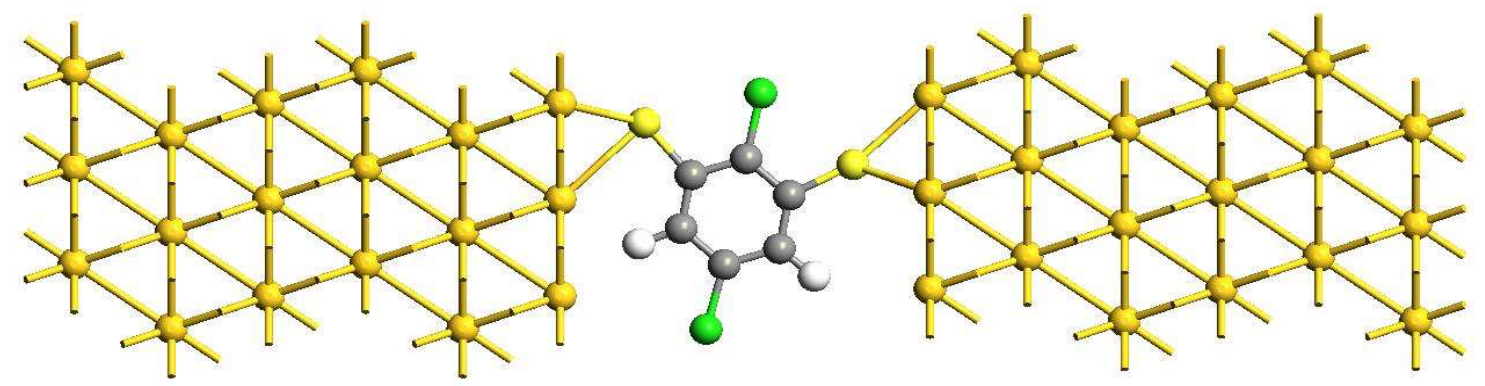

Fig.-1: Structure of Gold/2,5-dichlorobenzene-1,3-dithiol/gold Molecular Junction

\section{RESULTS AND DISCUSSION}

The structure of the 2,5-dichlorobenzene-1,3-dithiol molecule sandwiched at the hep site of gold electrodes was optimized using DFT with NEGF. Previously, we have reported for fcc site. ${ }^{30}$

The total density of states, projected device density of states, and transmission coefficient were calculated from $-3 \mathrm{eV}$ to $3 \mathrm{eV}$ using DFT with NEGF as mentioned in computational details.Fig.-2(a) shows the total density of states and projected density of states of the Gold/2,5-dichlorobenzene-1,3-dithiol/Gold junction at zero bias. In the projected density of states, two peaks were observed approximately at $1.8 \mathrm{eV}$ and $1 \mathrm{eV}$ in the HOMO region while in the LUMO region it was observed approximately at $2.9 \mathrm{eV}$. Almost at the same energy level, we could be able to observe peaks in the total density of states. This shows that the influence of a molecule in the total density of states. From Fig.-2(b) we observe that a greater number of peaks in the HOMO region are existing comparing to the LUMO region. This is due to the higher density of states in the HOMO region than LUMO.

We have plotted the transmission coefficient at $1.6 \mathrm{~V}$ in forward and reverse bias as shown in Fig.-3, since high rectification occurred at this bias. From this graph, we observed that the transmission coefficient near by the Fermi region is higher in the forward bias than the transmission coefficient in the reverse bias. Due to the change in the transmission coefficient, the current also gets changed when we switch it from forward to reverse bias. This change in current leads increases in the rectification ratio.

Figure-4(a) shows the current-voltage characteristic of the system at various applied bias which was calculated using the equation mentioned in computational details. We observed that the current increases in a linear way till $1 \mathrm{~V}$ at the forward bias further differential resistance effect was observed about $1 \mathrm{~V}$ to $2 \mathrm{~V}$ region. A similar kind of effect was observed in reverse bias. But in reverse bias, the differential resistance effect is small compared to the forward bias. This can be clearly seen in Fig.-4(b). The change in conductance is not just like a switching performance. This is due to resonant tunneling in the metalmolecular interface. From Fig.-4(c) it could be clearly observed that the conductance is very low in 
RASĀYAN J. Chem.

Vol. 13 | No. 3 |1412-1416| July - September | 2020

reverse bias compared to the forward bias. So, we predict that this molecule could exhibit a rectification effect. To confirm it we have plotted the rectification ratio for various applied bias (in Volts) as shown in fig. 5 . We observed that the rectification increases to $1.6 \mathrm{~V}$ further increasing the bias leads to a decrease in rectification. This type of change in the rectification effect already observed for organic molecules. ${ }^{31-33}$

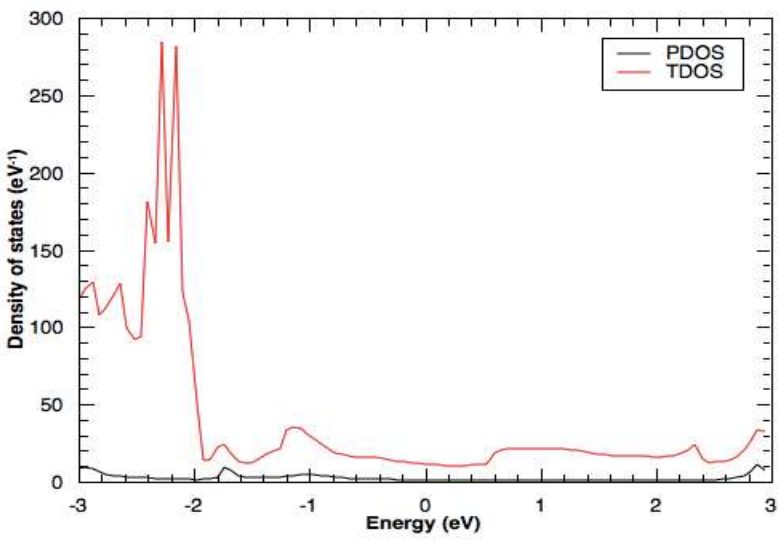

(a)

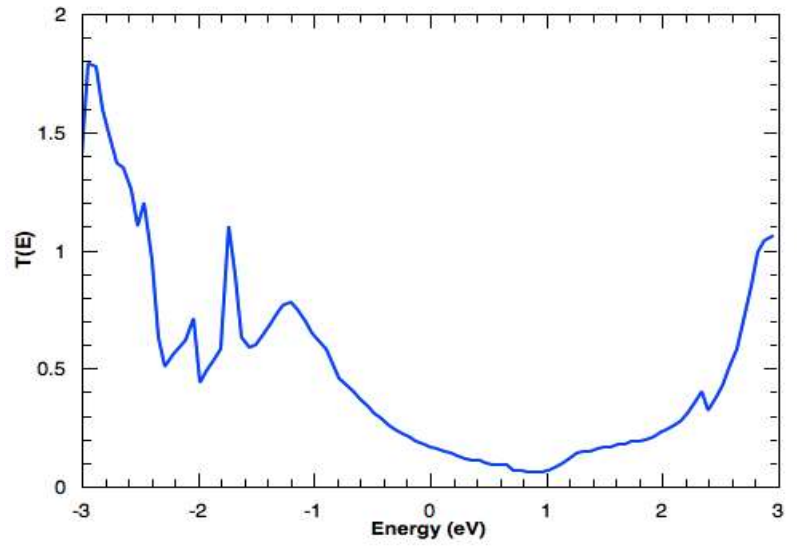

(b)

Fig.-2: (a) Total and Projected Density of States of Gold/2,5-dichlorobenzene-1,3-dithiol/gold Molecular Junction and (b)Transmission Coefficient of Gold/2,5-dichlorobenzene-1,3-dithiol/gold Molecular Junction at Zero Bias.

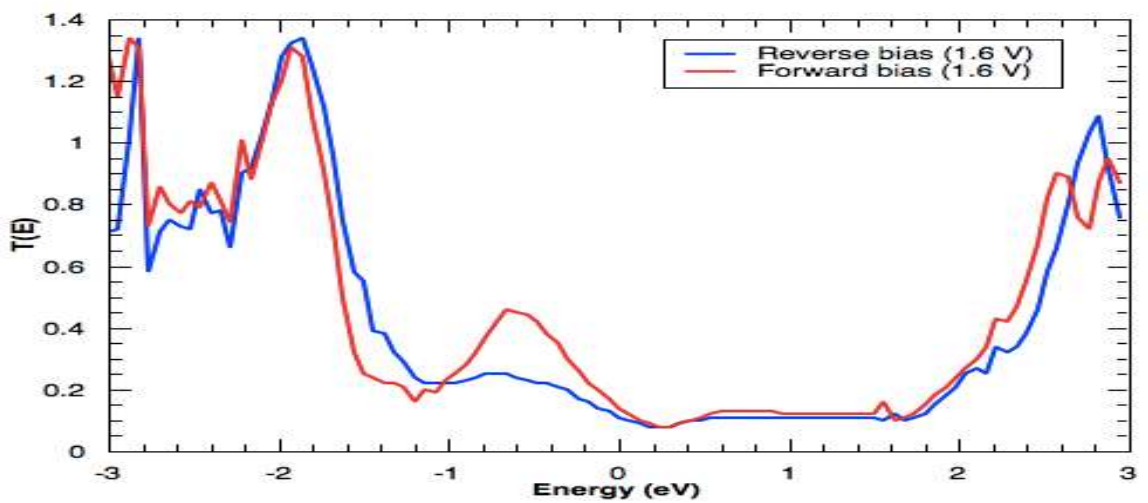

Fig.-3: Transmission Coefficient of Gold/2,5-dichlorobenzene-1,3-dithiol/gold Molecular Junction at 1.6 V

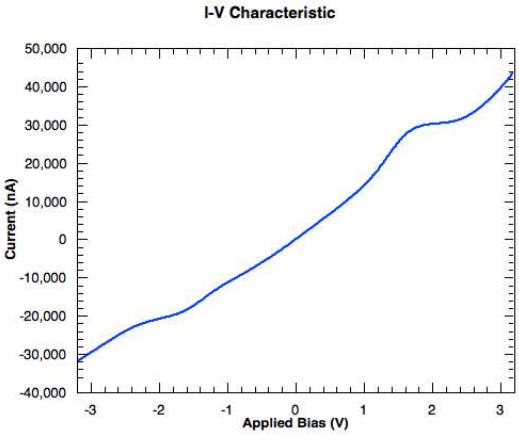

(a)

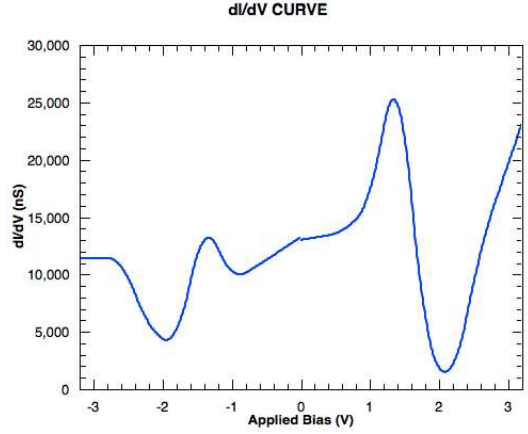

(b)

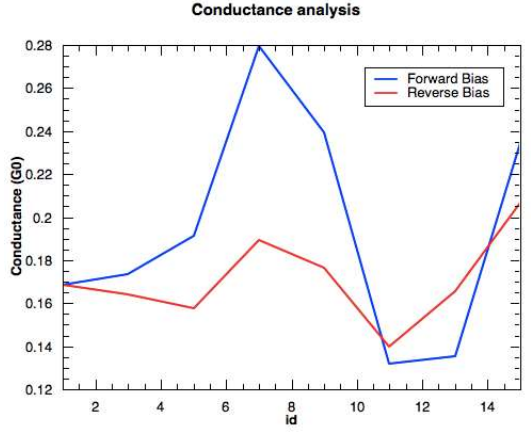

(c)

Fig.-4: (a)Current-voltage Characteristic, (b) dI/dV Curve and (c) Conductance of Gold/2,5-dichlorobenzene-1,3dithiol/gold Molecular Junction 
RASĀYAN J. Chem.

Vol. 13 | No. 3 |1412-1416| July - September | 2020

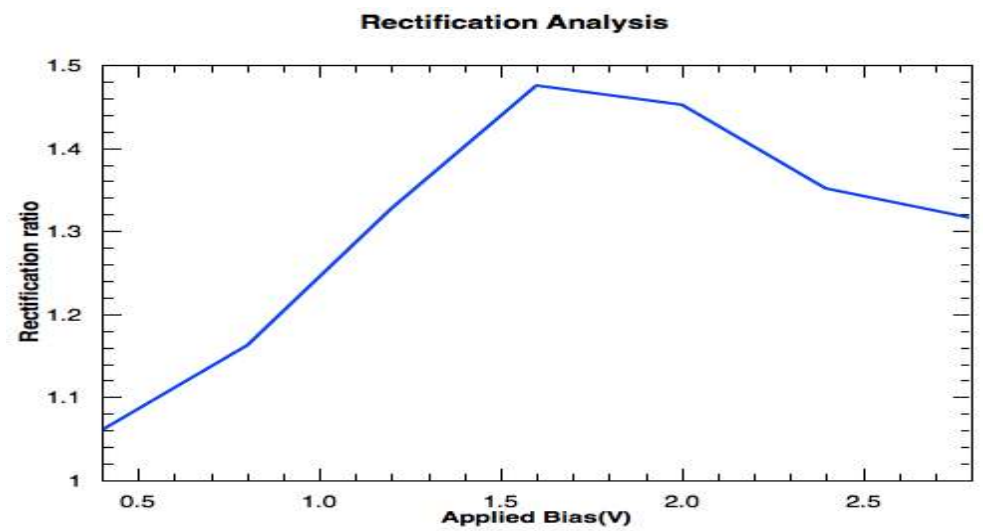

Fig.-5: Rectification Effect of Gold/2,5-dichlorobenzene-1,3-dithiol/gold Molecular Junction.

\section{CONCLUSION}

The electron transport properties of gold/2,5-dichlorobenzene-1,3-dithiol/gold molecular junction was studied using DFT with NEGF formalism. For that, we have computed the density of states (DOS), projected density of states (PDOS), transmission co-efficient, etc. From the density of states and projected density of states, we found that the molecule density of states influences the total density of states. At 1.6 $\mathrm{V}$ transmission coefficient is higher at nearby Fermi region in the forward bias comparing to reverse bias. Due to this the rectification ratio is high at this bias. Also, current-voltage characteristic shows differential resistance around $1 \mathrm{~V}$ to $2 \mathrm{~V}$. From this we conclude that the gold/2,5-dichlorobenzene-1,3-dithiol/gold molecular junction can be used in various electronic applications.

\section{ACKNOWLEDGMENT}

We gratefully acknowledge the financial support for this project from DST-FIST, Government of India (Ref.No SR/FST/PSI-155/2010).

\section{REFERENCES}

1. X.Zhang,T. Li, Chinese Chemical Letters, 28, 2058 (2017), DOI:10.1016/j.cclet.2017.09.008.

2. D.Vuillaume, ComptesRendus Physique, 9, 78 (2008), DOI:10.1016/j.crhy.2007.10.014.

3. C.Preferencial Kala, P.ArunaPriya, D.John Thiruvadigal, Nanoscience and Nanotechnology Letters, 1 (3), 224 (2009), DOI: 10.1166/nnl.2009.1033.

4. S.J.Higgins, R.J.Nichols, Polyhedron, 140, 25 (2018), DOI:10.1016/j.poly.2017.10.022.

5. T. Sebechlebská, J. Šebera, V. Kolivoška, M. Lindner, J. Gasior, G.Mészáros, M.Valášek, M. Mayor, M.Hromadová,Electrochimica Acta.,258, 1191(2017), DOI:10.1016/j.electacta.2017.11.174.

6. F.X. Zu, F.C. Zhang, D. Wang, Y. Ni, S.L. Wang, C.L. Ding, G.Y. Gao, Physics Letters A, 384, 126190 (2020), DOI:10.1016/j.physleta.2019.126190.

7. W.Kamiński, R.Topolnicki, P.Hapala, P.Jelínek, R.Kucharczyk, Organic Electronics: physics, materials, applications, 34, 254 (2016), DOI:10.1016/j.orgel.2016.04.035.

8. M.D.Ganji, F.Nourozi, Physica E: Low-Dimensional Systems and Nanostructures, 40, 2606 (2008), DOI:10.1016/j.physe.2007.09.123.

9. E.Leary, A.La Rosa, M.T.González, G.Rubio-Bollinger, N.Agraït, N.Martín,Chemical Society Reviews,44, 920 (2015), DOI:10.1039/C4CS00264D.

10. Y.Komoto, S.Fujii, M.Iwane, M.Kiguchi, Journal of Material Chemistry C, 4, 8842(2016), DOI:10.1039/C6TC03268K.

11. C. Jia, X.Guo, Chemical Society Reviews, 42, 5642 (2013), DOI:10.1039/c3cs35527f.

12. M. Kiguchi, O. Tal, S. Wohlthat, F. Pauly, M. Krieger, D. Djukic, J. C. Cuevas, J. M. Van Ruitenbeek,Physical Review Letters, 101, 046801 (2008), DOI:10.1103/PhysRevLett.101.046801.

13. S.Kaneko, T.Nakazumi, M.Kiguchi, Journal of Physical Chemistry Letters, 1, 3520(2010), DOI:10.1021/jz101506u.

14. M. Elbing, R. Ochs, M. Koentopp, M. Fischer, C. Von Hänisch, F. Weigend, F. Evers, H. B. Weber, M. Mayor, Proceedings of the National Academy of Sciences of the United States of America. 102, 8815 (2005), DOI:10.1073/pnas.0408888102. 
RASĀYAN J. Chem.

Vol. 13 | No. 3 |1412-1416| July - September | 2020

15. T.Ogawa, M.Handayani, Design Syntheses of Molecules for Nonlinear and Nonsymmetric SingleMolecule Electric Properties, pp. 419-437 (2017), DOI:10.1007/978-3-319-57096-9_17.

16. A.Aviram, M.A.Ratner, Chemical Physics Letters, 29, 277(1974), DOI:10.1016/00092614(74)85031-1.

17. J. Zhao, C. Zeng, X. Cheng, K. Wang, G. Wang, J. Yang, J. G. Hou, Q. Zhu, Physical Review Letters, 95, 045502 (2005), DOI:10.1103/PhysRevLett.95.045502.

18. R. M. Metzger, B. Chen, U. Höpfner, M. V. Lakshmikantham, D. Vuillaume, T. Kawai, X. Wu, H. Tachibana, T. V. Hughes, H. Sakurai, J. W. Baldwin, C. Hosch, M. P. Cava, L. Brehmer, G. J. Ashwell, Journal of the American Chemical Society, 119 , 10455 (1997), DOI:10.1021/ja971811e.

19. J. Hihath, C. Bruot, H. Nakamura, Y. Asai, I. Díez-Pérez, Y. Lee, L. Yu, N. Tao, ACS Nano, 5, 8331 (2011), DOI: 10.1021/nn2030644.

20. W.Kohn, L.J.Sham, Physical Review,140, A1133 (1965), DOI:10.1103/PhysRev.140.A1133.

21. J.D.Patterson, Annals of Nuclear Energy, 16, 611 (1989), DOI:10.1016/0306-4549(89)90016-9.

22. J. M. Soler, E. Artacho, J. D. Gale, A. García, J. Junquera, P. Ordejón, D. Sánchez-PortalJournal of Physics: Condensed Matter, 14, 2745 (2002), DOI:10.1088/0953-8984/14/11/302.

23. M.Brandbyge, J.L.Mozos, P.Ordejón, J.Taylor, K.Stokbro, Physical Review B - Condensed Matter and Materials Physics, 65, 1654011 (2002), DOI:10.1103/PhysRevB.65.165401.

24. K.Stokbro, J.Taylor, M.Brandbyge, P.Ordejón, Annals of the New York Academy of Sciences, 1006, 212 (2003), DOI: 10.1196/annals.1292.014.

25. J.P.Perdew, K.Burke, M.Ernzerhof, Physical Review Letters, 77, 3865(1996), DOI:10.1103/PhysRevLett.77.3865.

26. J.P.Perdew, K.Burke, M.Ernzerhof, Physical Review Letters, 78, 1396(1997), DOI:10.1103/PhysRevLett.78.1396.

27. R.P.Kaur, R.S.Sawhney, D.Engles, Molecular Physics, 114, 2289(2016), DOI:10.1080/00268976.2016.1197431.

28. Atomistix ToolKit version 12.8.2, QuantumWise A/S (www.quantumwise.com).

29. C.Cao, L.N.Chen, M.Q.Long, H.Xu, Physics Letters, Section A: General, Atomic and Solid State Physics, 377(31-33), 1905(2013), DOI:10.1016/j.physleta.2013.05.004.

30. A.Aadhityan, C.P. Kala, D.J.Thiruvadigal, AIP Conference Proceedings, 2005, 70001(2018), DOI:10.1063/1.5050758.

31. G.P.Zhang, Z.Xie,Y. Song, G.C.Hu, C.K.Wang, Chemical Physics Letters, 591, 296(2014), DOI:10.1016/j.cplett.2013.11.057.

32. Y.Song, D.L.Bao, Z.Xie, G.P.Zhang, C.K.Wang, Physics Letters, Section A: General, Atomic and Solid State Physics, 377, 3228 (2013), DOI:10.1016/j.physleta.2013.09.043.

33. M.L.Perrin, M.Doelman, R.Eelkema, H.S.J.VanDer Zant, Physical Chemistry Chemical Physics, 19, 29187 (2017), DOI:10.1039/C7CP04456A.

[RJC-3049/2019] 